\title{
Primary Preventive and Secondary Interventionary Effects of Acetyl-L-Carnitine on Diabetic Neuropathy in the Bio-Breeding Worcester Rat
}

\author{
Anders A.F. Sima, ${ }^{\star \S}$ Helen Ristic, ${ }^{\star \S}$ Andrew Merry, ${ }^{\star \S}$ Mikiko Kamijo, ${ }^{\star \S}$ Sarah A. Lattimer, ${ }^{\ddagger \S}$ Martin J. Stevens, ${ }^{\ddagger \S}$ \\ and Douglas A. Greene ${ }^{\ddagger \S}$ \\ Departments of *Pathology, ${ }^{\ddagger}$ Internal Medicine, and ${ }^{\S}$ Michigan Diabetes Research and Training Center, University of Michigan Medical \\ Center, Ann Arbor, Michigan 48109
}

\begin{abstract}
The abnormalities underlying diabetic neuropathy appear to be multiple and involve metabolic neuronal and vasomediated defects. The accumulation of long-chain fatty acids and impaired $\beta$-oxidation due to deficiencies in carnitine and/or its esterified derivatives, such as acetyl-L-carnitine, may have deleterious effects. In the present study, we examined, in the diabetic bio-breeding Worcester rat, the shortand long-term effects of acetyl-L-carnitine administration on peripheral nerve polyols, myoinositol, $\mathrm{Na}^{+} / \mathrm{K}^{+}$-ATPase, vasoactive prostaglandins, nerve conduction velocity, and pathologic changes. Short-term prevention (4 mo) with acetyl-L-carnitine had no effects on nerve polyols, but corrected the $\mathrm{Na}^{+} / \mathrm{K}^{+}$-ATPase defect and was associated with $63 \%$ prevention of the nerve conduction defect and complete prevention of structural changes. Long-term prevention $(8 \mathrm{mo}$ ) and intervention (from 4 to $8 \mathrm{mo}$ ) with acetyl-Lcarnitine treatment normalized nerve $\mathrm{PGE}_{1}$ whereas 6-keto $\mathrm{PGF}_{1 \alpha}$ and $\mathrm{PGE}_{2}$ were unaffected. In the prevention study, the conduction defect was $73 \%$ prevented and structural abnormalities attenuated. Intervention with acetyl-L-carnitine resulted in $76 \%$ recovery of the conduction defect and corrected neuropathologic changes characteristic of 4-mo diabetic rats. Acetyl-L-carnitine treatment promoted nerve fiber regeneration, which was increased two-fold compared to nontreated diabetic rats. These results demonstrate that acetyl-L-carnitine has a preventive effect on the acute $\mathrm{Na}^{+}$I $\mathrm{K}^{+}$-ATPase defect and a preventive and corrective effect on $\mathrm{PGE}_{1}$ in chronically diabetic nerve associated with improvements of nerve conduction velocity and pathologic changes. (J. Clin. Invest. 1996. 97:1900-1907.) Key words: neuropathy $\bullet$ acetyl-L-carnitine $\cdot$ nerve conduction velocity $\bullet$ morphometry $\bullet$ prostaglandins
\end{abstract}

\section{Introduction}

Human diabetic neuropathy is characterized by decreased nerve conduction velocity, vascular dysfunction, and progres-

This work was presented in part at the IVth Neurodiab Meeting, European Association for the Study of Diabetes, Düsseldorf, Germany, September 1994.

Address correspondence to Anders A.F. Sima, Wayne State University, 540 East Canfield Avenue, Detroit, MI 48201. Phone: 313577-1102; FAX: 313-577-0057.

Received for publication 23 June 1995 and accepted in revised form 1 February 1996.

J. Clin. Invest.

(C) The American Society for Clinical Investigation, Inc.

0021-9738/96/04/1900/08 \$2.00

Volume 97, Number 8, April 1996, 1900-1907 sive neuroanatomical changes affecting both neuronal structures, the endoneurial vasculature and supporting connective tissue elements (1-4). In animal models of diabetes mellitus, similar functional and structural changes have been associated with a number of metabolic abnormalities, all of which are believed to result from elevated glucose levels (5-8). One of the most thoroughly examined metabolic imbalances in peripheral nerve is the increased shunting of excessive glucose through the polyol pathway $(2,8-11)$, which is associated with myoinositol depletion and decreased protein kinase $\mathrm{C}(\mathrm{PKC})^{1}$ and $\mathrm{Na}^{+} / \mathrm{K}^{+}$-ATPase activities $(8,9)$. This cascade of abnormalities may affect the neuronal elements directly as well as the endoneurial vasculature (8). Other abnormalities include imbalances in the redox coupling of NADPH/NADP ${ }^{+}$and NADH/ $\mathrm{NAD}^{+}$associated with the reduction of glucose to sorbitol by aldose reductase and the oxidation of sorbitol to fructose by sorbitol dehydrogenase, respectively $(8,10)$. It has been suggested that imbalances in $\mathrm{NADPH} / \mathrm{NADP}^{+}$or $\mathrm{PKC}$ may result in decreased synthesis of NO via impaired NO-synthase activity resulting in vasoconstriction (8). Moreover, increased activity of sorbitol dehydrogenase, driven by polyol-pathway activation, will deplete $\mathrm{NAD}^{+}$and cause a shift in the lactate/ pyruvate ratio leading to a situation of "pseudohypoxia," (10) exacerbating the effects of true ischemia.

Other potentially contributory factors include glycation of structural and functional proteins, oxidative stress, and abnormalities in arachidonic acid metabolism, supported by positive treatment effects on diabetic neuropathy with aminoguanidine, antioxidants, and linolenic acid $(11,12)$. Impaired synthesis of vasoactive prostacyclins has been invoked in impaired endoneurial blood flow with associated slowing of nerve conduction velocity (NCV) (11). Vasoactive prostanoid analogues of $\mathrm{PGE}_{1}$ improve sciatic nerve blood flow and $\mathrm{NCV}$ in diabetic rats (13). Hence, endoneurial hypoxia is likely to constitute a major component in the pathogenesis of diabetic neuropathy with secondary adverse effects on neuronal function and structure. It is therefore becoming increasingly clear that diabetic neuropathy is a multifactorial disorder.

Carnitine and its short-chain esters facilitate transport of long-chain fatty acids across the inner mitochondrial membrane for $\beta$-oxidation, thereby promoting energy availability and preventing toxic accumulation of long-chain fatty acids (14). Recent studies have reported abnormal carnitine metabolism in diabetes (14-16). L-Carnitine (15) and acetyl-L-carnitine (ALC) (16) levels are decreased in sciatic nerve in streptozotosin rats, and ALC treatment corrects electroretinographic abnormalities, slowed nerve conduction velocity (15-

1. Abbreviations used in this paper: ALC, acetyl-L-carnitine; $\mathrm{BB} / \mathrm{W}$, bio-breeding Worcester; NCV, nerve condition velocity; PKC, protein kinase $\mathrm{C}$. 
17), and increased vascular permeability in ocular tissue and peripheral nerve (14).

We have previously reported on the metabolic, functional, and structural changes and their interrelationships in the spontaneously diabetic bio-breeding Worcester $(\mathrm{BB} / \mathrm{W})$ rat $(5,7$, 18-20). Structural changes, such as axonal atrophy and degeneration and nodal abnormalities, are present after 4 mo of diabetes and progress thereafter, culminating in a $50 \%$ myelinated fiber loss after 1 yr of diabetes $(5,20)$, similar to the progressive nerve fiber loss seen in human diabetic neuropathy $(1-3)$.

Pharmacological treatment of diabetic neuropathy will likely be used for intervention of subclinical or overt diabetic neuropathy. The present studies were therefore designed to assess the effects of both short- and long-term prevention, as well as intervention with ALC on the metabolic, functional, and structural abnormalities characterizing the neuropathy in the spontaneously diabetic $\mathrm{BB} / \mathrm{W}$ rat.

\section{Methods}

Animals. The present animal studies were approved by the Institutional Review Board of the University of Michigan. 34 prediabetic, diabetes-prone male $\mathrm{BB} / \mathrm{W}$ rats and 26 diabetes-resistant, agematched male BB/W rats were obtained from the National Institutes of Health-sponsored BB/W rat colony (Department of Pathology, University of Massachusetts, Worcester, MA). They were maintained in metabolic cages with ad lib. access to water and rat chow (5001; Ralston Purina Co., St. Louis, MO). Body weight, urine volume, and glucosuria (Ames Keto-Diastix; Miles Laboratories Inc., Research Products Div., Elkhart, IN) were monitored daily to ascertain onset of diabetes in diabetes-prone animals. After onset of diabetes, which occurred at $76 \pm 2 \mathrm{~d}$ of age, diabetic rats were supplemented with small doses $(0.5-3.0 \mu / \mathrm{d})$ of ultralente insulin (Novo Nordisk, Princeton, NJ) designed to maintain blood glucose levels between 15.0 and $25.0 \mathrm{mmol} /$ liter, and to prevent ketoacidosis. Blood glucose levels were measured biweekly in all rats and glycated hemoglobin on a monthly basis in 8-mo animals. The present examinations were divided into two independent studies. In the first study, diabetic (D4ALC) and age- and sex-matched control (C4ALC) animals were treated with ALC from onset of diabetes for $4 \mathrm{mo}$, and the data were compared with those obtained from nontreated control (C4) and diabetic animals (D4) (short-term prevention study). In the second study, control (C8ALC) and diabetic (D8ALC) rats were treated with ALC from onset of diabetes for 8 mo, and compared with nontreated control (C8) and diabetic (D8) animals (long-term prevention study). In this latter study, one group of diabetic rats was untreated from onset of diabetes up to $4 \mathrm{mo}$; ALC treatment was then initiated and continued for an additional 4 mo (D4-8ALC) (intervention study). Starting $1 \mathrm{wk}$ after onset of diabetes (prevention studies), or at 4 mo after detection (intervention study), animals were given daily doses of ALC (Alcar; Sigma Tau, S.p.a., Rome, Italy) (90-100 mg/kg body wt) by gavage.

Electrophysiologic studies. Baseline NCV was measured within $24 \mathrm{~h}$ of onset of diabetes, $1 \mathrm{wk}$ after onset of diabetes, before initiation of ALC treatment in the prevention groups, and thereafter every $4 \mathrm{wk}$. In the intervention group, NCV was obtained every $4 \mathrm{wk}$ and at 4 mo, just before starting ALC treatment. NCV was measured in the sciatic tibial conducting motor system under temperature controlled $\left(35^{\circ} \mathrm{C}-37^{\circ} \mathrm{C}\right)$ conditions using needle electrodes as previously described (19). Animals were anesthetized with diethyl ether (Fisher Scientific Co., Fair Lawn, NJ). The left sciatic nerve was stimulated with square pulses $(2 \mathrm{~Hz})$ at the sciatic notch, and the tibial nerve at the ankle by supramaximal $(8 \mathrm{~V})$ stimulations using an electromyography machine (5200A; Cadwell Laboratory Inc., Kennewick, WA). The compound-evoked motor responses, representing the averaging of 16 recordings, were obtained from the first interosseous muscle. $\mathrm{NCV}$ was calculated by subtracting the distal from the proximal latency (measured in milliseconds) divided into the distance (measured in millimeters) between the two stimulating electrodes, giving the $\mathrm{NCV}$ in $\mathrm{m} \cdot \mathrm{sec}^{-1}$.

Tissue collection. At the end of each study protocol, nonfasted animals were anesthetized with Na-pentobarbital $(50 \mathrm{mg} / \mathrm{kg}$ body wt i.p.). The left sciatic nerve was dissected and cleaned from surrounding tissues, weighed, and snap-frozen in liquid nitrogen and stored at $-70^{\circ} \mathrm{C}$. The animals were then perfused through the left heart with $\sim 500 \mathrm{ml}$ of $2.5 \%$ glutaraldehyde buffered with $0.1 \mathrm{M}$ cacodylate buffer at $\mathrm{pH}$ 7.40. The right sural nerve was dissected and postfixed in the same fixative for $2 \mathrm{~h}$ at $4^{\circ} \mathrm{C}$, and then further fixed in cacodylatebuffered $(0.1 \mathrm{M}) 1 \%$ osmium tetroxide $(\mathrm{pH} 7.40)$ for $2 \mathrm{~h}$ at $4^{\circ} \mathrm{C}$. The proximal sural nerve was dehydrated in ethanol, and cross sections were imbedded in Epon for light-microscopic morphometry. The distal sural nerve was dehydrated and used for teased fiber preparations in unpolymerized Epon as previously described (19).

Biochemical analyses. For the measurements of nerve glucose, sorbitol, fructose, and myoinositol, sciatic nerve samples were homogenized in $2 \mathrm{ml}$ of $5 \%$ TCA. Aldonitrile derivatives were formed by the addition of $0.3 \mathrm{ml}$ hydroxylamine in pyridine-methanol $4: 1$ (vol:vol). The samples were sonicated for $1 \mathrm{~min}$ and $1 \mathrm{ml}$ of acetic anhydride was added. After further sonication, $2 \mathrm{ml}$ of 1,2-dichlorothane was added and samples were washed in $1.0 \mathrm{~N}$ HCI. Before analysis, the samples were reconstituted in 2-butanane. The samples were analyzed in a gas-liquid chromatograph (3400; Varian; Sunnyvale, CA) as previously described (21).

$\mathrm{Na}^{+} / \mathrm{K}^{+}$-ATPase was measured as previously described (21). In brief, nerve samples were homogenized in $2 \mathrm{ml}$ of $0.2 \mathrm{M}$ sucrose plus $0.02 \mathrm{M}$ Tris- $\mathrm{HCl}$ at $\mathrm{pH} 7.5 .10-20 \mu \mathrm{l}$ of the homogenate was assayed enzymatically for total ATPase in $1 \mathrm{ml}$ of $100 \mathrm{mM} \mathrm{NaCl}, 10 \mathrm{mM} \mathrm{KCl}$, $2.5 \mathrm{mM} \mathrm{MgCl}_{2}, 1 \mathrm{mM}$ Tris ATP, $1 \mathrm{mM}$ phosphoenolpyruvate, $30 \mathrm{mM}$ imidazole-HCl buffer ( $\mathrm{pH}$ 7.3), $0.15 \mathrm{mM}$ NADH, $50 \mu \mathrm{g}$ lactate dehydrogenase, and $30 \mu \mathrm{g}$ pyruvate kinase. To measure ouabain-inhibitable ATPase $20 \mu \mathrm{l}$ of $25 \mathrm{mM}$ ouabain was added to each aliquot. Ouabain inhibitable $\mathrm{Na}^{+} / \mathrm{K}^{+}$-ATPase activity was defined as the difference in activity before and after the addition of ouabain and was expressed as micromoles ADP formed per gram of wet weight per hour.

$P G$ assessments. $\mathrm{PGE}_{2}$ and 6-keto- $\mathrm{F}_{1 \alpha}$ quantitations were assessed in sciatic nerve tissue harvested at death and stored $(2 \mathrm{wk}-4$ $\mathrm{mo})$ at $-70^{\circ} \mathrm{C}$ until assay. Tissue was ground with a glass homogenizer (Thomas Scientific, Swedesboro, NJ) in cold $\left(4^{\circ} \mathrm{C}\right)$ PBS $(0.001 \%$ BSA, $\mathrm{pH}$ 7.4) and assessed for PG content by competitive $\left(\mathrm{E}_{2}\right)$ and noncompetitive (6-keto- $\mathrm{F}_{1 \alpha}$ ) acetylcholinesterase-based ELISA systems (ACE ${ }^{\mathrm{TM}}$ EIA; Cayman Chem Co. Inc., Ann Arbor, MI) and read spectrophotometrically (SLT Labinstruments GmbH, Salzburg, Austria). $\mathrm{PGE}_{1}$ was quantitated using the same homogenates. A polyclonal anti- $\mathrm{E}_{1}$ serum (PerSeptive Biosystems, Cambridge, MA) was applied using an indirect alkaline phosphotase-based ELISA. Antigen was bound to 96-well plates using borate-binding buffer, $\mathrm{pH}$ 9.0. $\mathrm{PGE}_{1}$ levels were read spectrophotometrically. Quantitations were expressed as picograms of the relevant PG per mg wet weight of sciatic nerve. To test the cross-reactivity of $\mathrm{PGE}_{1}$ and $\mathrm{PGE}_{2}$ antibodies, HPLC-separated $\mathrm{PGE}_{1}$ and $\mathrm{PGE}_{2}$ fractions were tested separately and compared to their admixture with the two antibodies and the percentage cross-reactivity was calculated. For $\mathrm{PGE}_{1}$ it was $6.0 \%$ and for $\mathrm{PGE}_{2}, 7.8 \%$.

Morphometric analyses. Semithin $(0.75-\mu \mathrm{m})$ cross sections of the sural nerve were stained with paraphenylene diamine for light microscopic morphometric analysis using a computerized image analysis system (Image-1; Universal Imaging Corp., West Chester, PA). The data were compiled using a 486 personal computer compatible with 8 MB RAM. This system allows for assessment of the total complement of sural nerve myelinated fibers and provides the following measures: mean myelinated fiber size $\left(\mu \mathrm{m}^{2}\right)$, myelinated fiber density $\left(\# / \mathrm{mm}^{2}\right)$, coefficient of variation of fiber densities between image frames, myelinated fiber occupancy (percentage of endoneurial area occupied 
by myelinated fibers), and axon/myelin ratio, as previously described (19).

Teased fiber evaluation. A mean of $158 \pm 2$ myelinated fibers were teased from each sural nerve and scored for specific pathologies, providing a more sensitive method compared to light-microscopic morphometric analysis (19). The pathologic changes seen in diabetic neuropathy evolve from mild abnormalities to changes of increasing severity in the following order: normality, paranodal swelling, paranodal demyelination, excessive myelin wrinkling, intercalated internodes, segmental demyelination, and remyelination/regeneration and Wallerian degeneration. Each fiber was scored according to its most severe change, as previously defined in detail (19). All teased fiber abnormalities except regenerated fibers were expressed as a percentage of total fibers minus regenerated fibers, in order not to diminish the frequencies of pathologic changes in ALC-treated animals, in which there was an increased number of regenerated fibers. The frequency of regenerated fibers was expressed as a percentage of total fibers.

Statistical evaluation. All data are expressed as mean \pm SEM. Significance of differences was calculated by ANOVA, and Scheffe's modified $t$ test. All measurements were performed by investigators who were unaware of the source of the tissues.

\section{Results}

\section{Body weight and hyperglycemia (Table I)}

Diabetic animals at both 4 and 8 mo of diabetes showed decreased body weights and increased blood sugar and glycated hemoglobin levels (Table I). ALC treatment had no effects on body weight gain or hyperglycemia in diabetic or control rats (Table I).

\section{The effect of ALC treatment on NCV (Fig. 1, $a$ and $b$ )}

In both the 4 and 8 mo experiments, diabetic nontreated animals showed a significant $(P<0.001) 18 \%$ slowing in NCV at 1 wk of diabetes. In 4 mo ALC-treated diabetic animals, there

Table I. Body Weights, Blood Glucose, and Glycated Hemoglobin Levels in Control, Diabetic, and Control and Diabetic ALC-treated Animals at 4 mo and 8 mo

\begin{tabular}{lcrc}
\hline \multicolumn{1}{c}{ Animals } & $\begin{array}{c}\text { Body } \\
\text { weight }\end{array}$ & \multicolumn{1}{c}{$\begin{array}{c}\text { Blood } \\
\text { glucose }\end{array}$} & Gly Hb \\
\hline & grams & mmol/liter & $\%$ \\
4 mo & & & \\
C4 $(n=5)$ & $454.4 \pm 10.2$ & $5.7 \pm 0.3$ & N/A \\
C4ALC $(n=5)$ & $443.8 \pm 7.4$ & $5.9 \pm 0.3$ & N/A \\
D4 $(n=5)$ & $305.8 \pm 11.8$ & $18.9 \pm 1.1$ & N/A \\
D4ALC $(n=5)$ & $323.8 \pm 10.3$ & $19.5 \pm 1.2$ & N/A \\
8 mo & & & \\
C8 $(n=8)$ & $500.5 \pm 6.0$ & $5.7 \pm 0.4$ & $5.6 \pm 0.2$ \\
C8ALC $(n=8)$ & $492.0 \pm 8.6$ & $5.9 \pm 0.3$ & $5.2 \pm 0.3$ \\
D8 $(n=9)$ & $358.2 \pm 11.6$ & $16.8 \pm 1.3$ & $10.3 \pm 0.8$ \\
D8ALC $(0-8$ mo $)(n=9)$ & $338.1 \pm 9.1$ & $16.6 \pm 0.7$ & $12.7 \pm 0.6$ \\
D4-8ALC $(4-8$ mo $)$ & $350.3 \pm 7.9$ & $16.3 \pm 1.2$ & $11.0 \pm 1.3$ \\
$\quad(n=6)$ & & & \\
& & &
\end{tabular}

C4, 4 mo nontreated control rats; C4ALC, 4 mo ALC-treated control rats; D4, 4 mo nontreated diabetic rats; D4ALC, 4 mo ALC-treated diabetic rats; $\mathrm{C} 8,8$ mo nontreated control rats; C8ALC, 8 mo ALC-treated control rats; D8, 8 mo nontreated diabetic rats; D8ALC, 8 mo ALCtreated diabetic rats; D4-8ALC, 8 mo diabetic rats treated with ALC from 4 to 8 mo of diabetes; N/A, not assessed. was a complete prevention of the NCV defect up to $4 \mathrm{wk}$ of diabetes (Fig. $1 \mathrm{a}$ ). At 4 mo, there was a significant $(P<0.001)$ $(63 \%)$ prevention of the NCV defect, leaving a small but significant $(P<0.002)$ residual NCV defect compared to nondiabetic control rats (Fig. $1 a$ ). ALC treatment of control rats had no effect on NCV.

8 mo nontreated diabetic animals showed, at $4 \mathrm{mo}$, an NCV slowing that was similar to that of nontreated diabetic rats in the 4 mo experiment (Fig. $1 \mathrm{~b}$ ). In diabetic rats treated with ALC from onset of diabetes, the NCV was, at 8 mo, $92 \%$ of that of controls; whereas, in 8 mo nontreated diabetic rats, the NCV was $70 \%$ of control values, resulting in a $73 \%(P<$ $0.001)$ protection of the NCV defect in ALC-treated rats.

\section{a Sciatic Nerve Conduction Velocity in $\mathrm{BB} / \mathrm{W}$-rats during 4 mo ALC treatment}
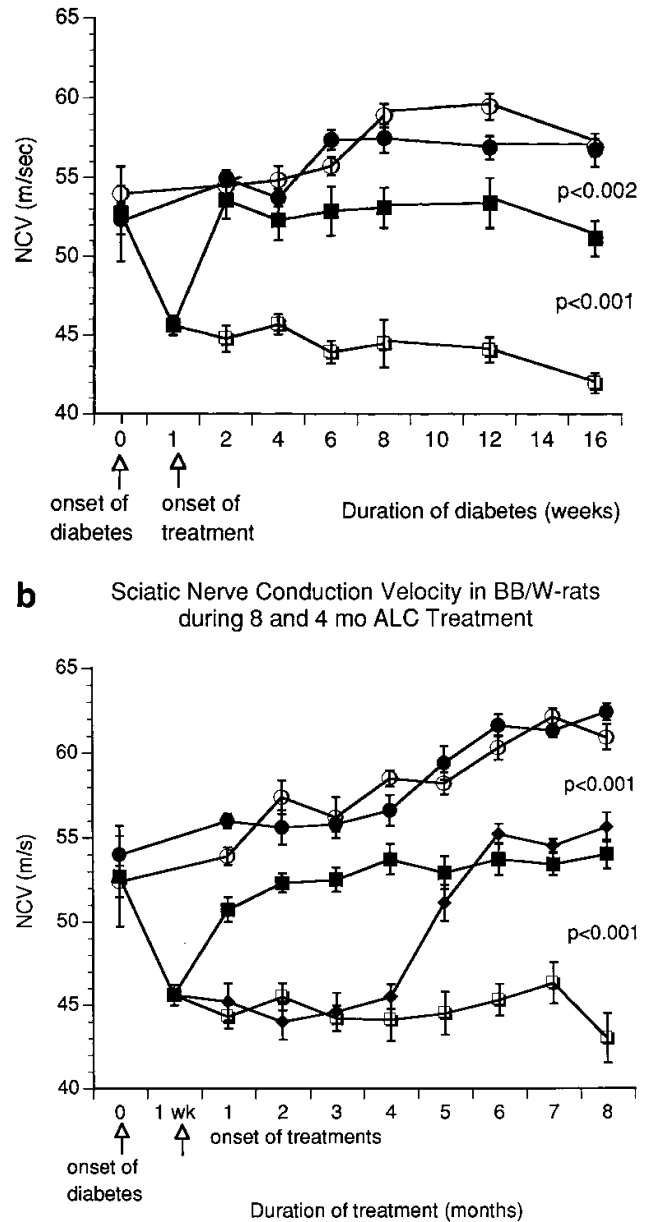

Figure 1. (a) NCV was measured within $24 \mathrm{hr}$ of onset of diabetes. These measurements served as baseline $\mathrm{NCV}$ at time point 0 . NCV velocities were again obtained $1 \mathrm{wk}$ after onset of diabetes just prior to initiation of treatment. NCVs were then measured on a biweekly basis up to the end of study protocol at $4 \mathrm{mo}$. Designation of animals into the various groups is the same as in Table $1 . \bigcirc, \mathrm{C} 4(n=7) ; \bullet$, C4ALC $(n=8)$; $\square$, D4 $(n=7) ; \boldsymbol{\square}$, D4ALC $(n=6)$. (b) NCV was measured within $24 \mathrm{~h}$ of onset of diabetes and these data served as the baseline (time-point 0 ). NCVs were again measured just before onset of ALC treatment at $1 \mathrm{wk}$ (prevention group) and 4 mo (intervention group). In addition, NCVs were measured longitudinally every month till the end of the study protocols at 8 mo of diabetes. Designation of animal groups is the same as in Table I. $\bigcirc, \mathrm{C} 8(n=8)$; $\bullet$, $\operatorname{C8ALC}(n=8) ; \square, \operatorname{D8}(n=5) ; \boldsymbol{\square}, \operatorname{D8ALC}(n=9) ; \bullet, \operatorname{D} 4-8 \operatorname{ALC}(n=6)$. 
Table II. The Effect of 4 and 8 mo ALC Treatments on Sciatic Nerve Glucose, Sorbitol, Fructose and Myoinositol Levels, and $\mathrm{Na}^{+} / \mathrm{K}^{+}$-ATPase Activity in Sciatic Nerves

\begin{tabular}{|c|c|c|c|c|c|}
\hline Animals & Glucose & Sorbitol & Fructose & Myoinositol & $\mathrm{Na}^{+} / \mathrm{K}^{+}$-ATPase \\
\hline & \multicolumn{4}{|c|}{$n m o l / m g$ wet $w t$} & $\mu m o l$ ADP/mg wet wt per $h$ \\
\hline \multicolumn{6}{|l|}{$4 \mathrm{mo}$} \\
\hline $\mathrm{C} 4(n=6)$ & $0.71 \pm 0.13$ & $0.17 \pm 0.02$ & $0.39 \pm 0.05$ & $3.27 \pm 0.24$ & $161.8 \pm 6.4 *$ \\
\hline $\operatorname{C4ALC}(n=6)$ & $0.59 \pm 0.21$ & $0.14 \pm 0.01$ & $0.34 \pm 0.04$ & $3.16 \pm 0.21$ & $143.7 \pm 10.4^{\ddagger}$ \\
\hline $\mathrm{D} 4(n=4)$ & $3.75 \pm 0.39^{\S}$ & $0.59 \pm 0.10^{\|}$ & $1.68 \pm 0.40^{\|}$ & $2.90 \pm 0.14$ & $128.6 \pm 5.8$ \\
\hline D4ALC $(n=4)$ & $2.82 \pm 1.25^{\S}$ & $0.50 \pm 0.30$ & $1.42 \pm 0.99$ & $3.12 \pm 0.19$ & $159.3 \pm 8.1^{\llbracket}$ \\
\hline \multicolumn{6}{|l|}{$8 \mathrm{mo}$} \\
\hline $\mathrm{C} 8(n=5)$ & $0.63 \pm 0.22$ & $0.04 \pm 0.01$ & $0.90 \pm 0.18$ & $1.87 \pm 0.16$ & $97.0 \pm 4.2$ \\
\hline $\operatorname{C8ALC}(n=8)$ & $0.68 \pm 0.13$ & $0.05 \pm 0.01$ & $0.74 \pm 0.15$ & $2.15 \pm 0.26$ & $105.2 \pm 8.7$ \\
\hline D8 $(n=8)$ & $7.19 \pm 1.24 * *$ & $0.37 \pm 0.07^{\text {辣 }}$ & $7.76 \pm 1.91^{\text {辛 }}$ & $1.77 \pm 0.20$ & $85.3 \pm 9.4$ \\
\hline $\operatorname{D8ALC}(n=9)$ & $8.77 \pm 1.14 * *$ & $0.31 \pm 0.07^{\text {螼 }}$ & $6.34 \pm 0.92^{\ddagger \ddagger}$ & $1.83 \pm 0.18$ & $89.9 \pm 7.4$ \\
\hline D4-8ALC $(n=5)$ & $5.69 \pm 1.41 * *$ & $0.33 \pm 0.09^{\text {辛 }}$ & 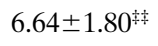 & $1.78 \pm 0.36$ & $86.5 \pm 8.4$ \\
\hline
\end{tabular}

Designation of animal groups as in Table $1 .{ }^{\S} P<0.01, \| P<0.05$ vs $\mathrm{C} 4$ and $\mathrm{C} 4 \mathrm{ALC}$ groups; ${ }^{*} P<0.002,{ }^{\ddagger} P<0.04,{ }^{\mathbb{}} P<0.003$ all vs D4; $* * P<0.006$ vs $\mathrm{C} 8$ and C8ALC groups; ${ }^{*} P<0.01$ vs C8 and C8ALC groups.

However, a small but significant $(P<0.001)$ NCV defect remained in ALC-treated rats (Fig. $1 b$ ). Intervention with ALC from 4-8 mo of diabetes resulted in a significant $(P<0.001)$ $76 \%$ recovery of the NCV defect (Fig. $1 b$ ). ALC treatment had no effect on NCV in nondiabetic control rats.

\section{The effect of ALC treatment on polyols and $\mathrm{Na}^{+} / \mathrm{K}^{+}-A T P a s e$ (Table II)}

ALC treatment had no effect on the elevated nerve glucose, sorbitol, or fructose levels in 4 mo treated diabetic animals, nor did ALC treatment have an effect on these parameters in control animals (Table II). Sciatic nerve myoinositol levels were not significantly altered by diabetes or ALC treatment (Table II).

Table III. The Effect of Diabetes and ALC Treatment on Nerve PGs

\begin{tabular}{lccc}
\hline \multirow{2}{*}{ Animals } & \multicolumn{3}{c}{ Prostaglandins } \\
\cline { 2 - 4 } & PGE $_{1}$ & PGE $_{2}$ & PGF $_{1 \alpha}$ \\
\hline \multicolumn{3}{c}{$p g / m g$ sciatic nerve } \\
8 mo & & \\
C8 $(n=8)$ & $1,737 \pm 233$ & $1,055 \pm 136$ & $668 \pm 90$ \\
C8ALC $(n=8)$ & $1,611 \pm 292$ & $467 \pm 194^{*}$ & $375 \pm 57^{\ddagger}$ \\
D8 $(n=9)$ & $702 \pm 155^{\S \|}$ & $192 \pm 138^{\sharp}$ & $210 \pm 28^{\ddagger * *}$ \\
D8 ALC $(n=9)$ & $1,816 \pm 405$ & $157 \pm 96^{\star}$ & $195 \pm 30^{\ddagger * *}$ \\
D4-8 ALC $(n=6)$ & $1,994 \pm 229$ & $209 \pm 130^{\sharp}$ & $178 \pm 34^{\ddagger * *}$ \\
& & &
\end{tabular}

Sciatic nerve PGs $\mathrm{PGE}_{1}, \mathrm{PGE}_{2}$, and 6-keto-PGF ${ }_{1 \alpha}$ were assessed by ELISA methods in 8 mo control and diabetic rats. PGE $_{1}$ was not effected by ALC treatment in control rats (C8ALC), but was significantly decreased in nontreated diabetic rats (D8) and restored in ALC-treated diabetic rats (D8ALC and D4-8ALC). $\mathrm{PGE}_{2}$ and $\mathrm{PGF}_{1 \alpha}$ were significantly decreased in ALC-treated control rats (C8ALC) and diabetic (D8) rats compared to nontreated control rats (C8). ALC treatment (D8ALC and D4-8ALC) had no effect on the decreased $\mathrm{PGE}_{2}$ and $\mathrm{PGF}_{1 \alpha}$ levels in diabetic rats. Designation of animal groups as in Table I. ${ }^{\S} P<0.05$ vs $\mathrm{C} 8$ and C8ALC; $\| P<0.05$ vs D8ALC and D4-8ALC; ${ }^{\pi} P<$ 0.001 vs $\mathrm{C} 8 ; * P<0.01$ vs $\mathrm{C} 8 ;{ }^{*} P<0.001$ vs $\mathrm{C} 8 ; * * P<0.03$ vs $\mathrm{C} 8 \mathrm{ALC}$.
In contrast, 4 mo of ALC treatment resulted in a complete prevention of the characteristic $\mathrm{Na}^{+} / \mathrm{K}^{+}$-ATPase defect, which was decreased by $\sim 20 \%(P<0.002)$ in nontreated diabetic rats (Table II). Treatment with ALC had no significant effect on $\mathrm{Na}^{+} / \mathrm{K}^{+}$-ATPase activity in nondiabetic rats.

In 8 mo diabetic animals, nerve glucose and fructose levels were significantly $(P<0.005)$ increased over those in 4 mo diabetic animals. On the other hand, sorbitol levels as well as $\mathrm{Na}^{+} / \mathrm{K}^{+}$-ATPase activities were decreased in both nontreated control and diabetic animals at 8 mo compared to those at 4 mo. ALC treatment had no effect of nerve polyol levels or $\mathrm{Na}^{+} / \mathrm{K}^{+}$-ATPase activities in 8 mo diabetic or control animals (Table II).

Table IV. The Effect of 4 and 8 Mo ALC Treatment on Myelinated Fiber Size, Fiber Density, and Axon/Myelin Ratio

\begin{tabular}{lccc}
\hline \multicolumn{1}{c}{ Animals } & $\begin{array}{c}\text { Myelinated } \\
\text { fiber size }\end{array}$ & $\begin{array}{c}\text { Myelinated } \\
\text { fiber density }\end{array}$ & $\begin{array}{c}\text { Axon/myelin } \\
\text { ratio }\end{array}$ \\
\hline & $\mu m^{2}$ & $N o . \mu m^{2}$ & $\mu m^{2} / \mu m^{2}$ \\
C8 $(n=4)$ & $44.9 \pm 1.3$ & $12,960 \pm 347^{*}$ & $0.619 \pm 0.003^{\ddagger}$ \\
C8ALC $(n=4)$ & $40.6 \pm 1.2$ & $13,934 \pm 178^{*}$ & $0.590 \pm 0.014^{\ddagger}$ \\
D8 $(n=5)$ & $37.3 \pm 1.2^{\S}$ & $12,778 \pm 359^{*}$ & $0.577 \pm 0.008$ \\
D8ALC $(n=5)$ & $32.5 \pm 0.8^{\| \uparrow * *}$ & $15,343 \pm 334$ & $0.609 \pm 0.006^{\ddagger}$ \\
D4-8ALC $(n=4)$ & $40.1 \pm 2.5^{\ddagger \ddagger}$ & $13,962 \pm 327$ & $0.612 \pm 0.005^{\ddagger}$ \\
& & & \\
\hline
\end{tabular}

In 8 mo diabetic rats, mean myelinated fiber size was significantly decreased compared to control (C8) rats. ALC treatment for $8 \mathrm{mo}$ (D8ALC) resulted in further significant decrease in fiber size. This effect, which can be accounted for by the addition of small regenerated fibers, was not evident in diabetic rats treated from 4 to 8 mo of diabetes (D4-8ALC). Myelinated fiber density was not decreased in diabetic rats (D8). ALC treatment (D8ALC) resulted in a small but significant increase in fiber density, probably related to the twofold increase in regenerated fibers in this group. A similar effect was not obvious in the intervention group (D4-8ALC). Axon/myelin ratio was significantly decreased in nontreated diabetic rats (D8), reflecting axonal atrophy. This abnormality was prevented (D8ALC) and corrected (D4-8ALC) in ALC-treated rats. Symbols for animal groups as in Table I. ${ }^{\sharp} P<0.04$ vs C 8 ; ${ }^{\S} P<0.002$ vs C8; $\| P<0.001$ vs C8ALC; $" P<0.03$ vs D8; ** $P<0.02$ vs D4-8ALc; $* P<0.05$ vs D8ALC; ${ }^{*} P<0.05$ vs D8. 


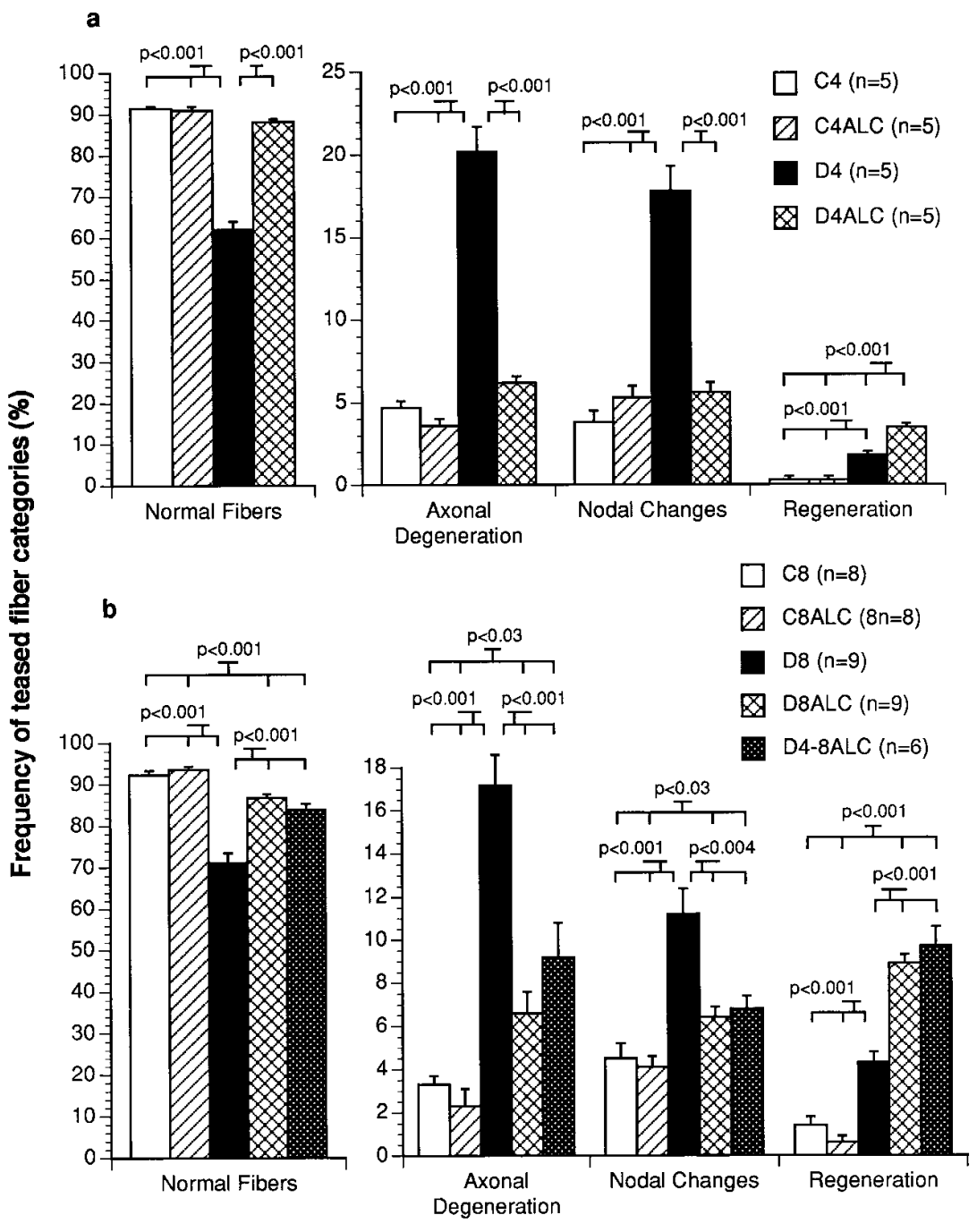

Figure 2. (a) The frequency of normal teased fibers was significantly decreased in nontreated diabetic (D4) rats compared to control (C4 and C4ALC) and ALC-treated (D4ALC) rats. This corresponded to significantly increased frequencies of fibers showing axonal degeneration and nodal changes in D4 rats, changes which were completely prevented in D4ALC rats. ALCtreated rats (D4ALC) showed significantly $(P<$ $0.001)$ increased frequencies of regenerated fibers. (b) 8 mo nontreated diabetic rats (D8) showed significantly decreased frequencies of normal fibers, and increased frequencies of fibers demonstrating axonal degeneration and nodal changes compared to controls (C8 and C8ALC) and ALC-treated (D8ALC and D4-8ALC) rats. However, these latter groups showed partial prevention (D8ALC) and correction (D4-8ALC) of axonal and nodal structural defects leaving small $(P<0.03)$ residual defects. Both D8ALC and D4-8ALC rats showed significantly increased $(P<0.001)$ frequencies of regenerating fibers.

\section{Effect of ALC treatment on sciatic nerve PGs (Table III)}

The $\mathrm{PGH}_{2}$ derived 6-keto- $\mathrm{PGF}_{1 \alpha}$ and $\mathrm{PGE}_{2}$, as well as the $\gamma$-linolenic acid derived $\mathrm{PGE}_{1}$ were significantly (at least $P<0.05$ ) decreased in nontreated 8 mo diabetic rats (Table III). ALC treatment in both D8ALC and D4-8ALC animals normalized sciatic nerve $\mathrm{PGE}_{1}$ levels, but had no effect on 6-keto-PGF ${ }_{1 \alpha}$ or $\mathrm{PGE}_{2}$ levels (Table III). Interestingly, ALC treatment lowered both 6-keto-PGF ${ }_{1 \alpha}(P<0.001)$ and $\mathrm{PGE}_{2}(P<0.01)$ levels in ALC-treated control rats. Sciatic nerve $\mathrm{PGE}_{1}$ levels were not effected by ALC in control rats (Table III).

\section{Effect of ALC treatment on myelinated fiber morphometry (Table IV)}

4 mo. Diabetes had no effect on myelinated fiber density, occupancy, coefficient of variation of fiber density, or axon-myelin ratio in the sural nerve, nor did ALC treatment have any effect on these parameters (data not shown). In contrast, there was a small $(14 \%, P<0.05)$ decrease in mean myelinated fiber size, which was completely prevented by ALC treatment.

8 mo. Mean fiber size was significantly $(P<0.002)$ decreased in 8 mo nontreated diabetic rats compared to control rats. Rats treated with ALC from onset of diabetes showed a mean fiber size significantly $(P<0.03)$ less than that of nontreated diabetic rats; whereas, those treated from 4-8 mo showed a mean fiber size less than that of control rats $(P<$ $0.04)$, but not significantly different from nontreated diabetic rats (Table IV). Nontreated diabetic and control rats showed no difference in myelinated fiber density. However, myelinated fiber density in diabetic rats treated with ALC for 8 mo showed an $18 \%(P<0.05)$ increase in fiber density compared to control rats (Table IV). Diabetic rats treated from 4-8 mo showed a nonsignificant $8 \%$ increase in fiber density compared to control rats. Nontreated diabetic rats showed a significant $(P<0.05) 7 \%$ decrease in axon-myelin ratio reflecting axonal atrophy. This defect was fully prevented or corrected after ALC treatment of diabetic rats.

Teased-fiber assessments after ALC treatment (Fig. 2, a and b; Tables $V$ and $V I$ )

The teased-fiber data are presented as frequencies of structurally normal fibers, the composite frequencies of axonal degeneration (excessive myelin wrinkling plus Wallerian degeneration), nodal degenerative changes (paranodal swelling plus paranodal demyelination plus intercalated internodes), and regenerated fibers (Fig. 2, $a$ and $b$ ). The frequencies of specific axonal and nodal changes are presented in Tables $\mathrm{V}$ and VI.

4 mo. Diabetic animals showed a significant $(P<0.001)$ $33 \%$ decrease in normal fibers, which was due to significant in- 
Table $V$. The Effect of 4 and 8 mo ALC Treatments on Excessive Myelin Wrinkling Reflecting Axonal Atrophy and Wallerian Degeneration

\begin{tabular}{lcc}
\hline \multicolumn{1}{c}{ Animals } & $\begin{array}{c}\text { Excessive } \\
\text { myelin wrinkling }\end{array}$ & $\begin{array}{c}\text { Wallerian } \\
\text { degeneration }\end{array}$ \\
\hline 4 mo & & \\
C4 $(n=5)$ & $4.6 \pm 0.5$ & $0.1 \pm 0.1$ \\
C4ALC $(n=5)$ & $3.6 \pm 0.4$ & 0 \\
D4 $(n=5)$ & $19.7 \pm 1.7^{*}$ & $0.5 \pm 0.2^{\ddagger}$ \\
D4ALC $(n=5)$ & $5.9 \pm 0.5^{\S}$ & $0.3 \pm 0.2$ \\
8 mo & & \\
C8 $(n=8)$ & $3.0 \pm 1.1$ & $0.3 \pm 0.1$ \\
C8ALC $(n=8)$ & $1.4 \pm 0.5$ & $0.8 \pm 0.3$ \\
D8 $(n=9)$ & $14.8 \pm 1.3^{\|}$ & $2.4 \pm 0.4^{4 * *}$ \\
D8ALC $(n=9)$ & $5.0 \pm 0.7^{\ddagger \ddagger}$ & $1.7 \pm 0.4^{4}$ \\
D4-8ALC $(n=6)$ & $6.9 \pm 1.2^{\S \ddagger} \S \S$ & $2.3 \pm 0.7 \pi * *$ \\
& & \\
\hline
\end{tabular}

Designation of animal groups as in Table I. $* P<0.001$ vs all groups; ${ }^{*} P<$ 0.03 vs C4ALC; ${ }^{P} P<0.001$ vs $C 8 ;{ }^{\ddagger} P<0.005$ vs C8ALC; ${ }^{\circledR} P<0.001$ vs D8; ${ }^{\S \S} P<0.006$ vs C8ALC; ${ }^{\circledR} P<0.01$ vs $\mathrm{C} 8$; $* * P<0.02$ vs C8ALC.

creases in fibers exhibiting axonal degeneration $(P<0.001)$, nodal degenerative changes $(P<0.001)$, and fiber regeneration $(P<0.001)($ Fig. $2 a)$. Axonal and nodal degenerative changes were completely prevented by 4 mo ALC treatment. Since Wallerian degeneration was not affected by ALC treatment (Table $\mathrm{V}$ ), the preventive effect of axonal degenerative changes was mainly due to prevention of axonal atrophy $(P<$ 0.001 ) reflected by the frequency of fibers exhibiting excessive myelin wrinkling (Table V). In 4 mo nontreated diabetic rats, all three sequential nodal changes (paranodal swelling, paranodal demyelination, and intercalated internodes) were significantly $(P<0.001)$ increased and completely prevented by ALC treatment (Table VI). Myelinated fiber regeneration showed a small but significant $(P<0.001)$ increase in non-

Table VI. The Effect of 4 and 8 mo ALC Treatments on Detailed Nodal Changes

\begin{tabular}{lccc}
\hline \multicolumn{1}{c}{ Animals } & $\begin{array}{c}\text { Paranodal } \\
\text { swelling }\end{array}$ & $\begin{array}{c}\text { Paranodal } \\
\text { demyelination }\end{array}$ & $\begin{array}{c}\text { Intercalated } \\
\text { nodes }\end{array}$ \\
\hline 4 mo & & & \\
C4 $(n=5)$ & $2.3 \pm 0.2$ & $0.9 \pm 0.2$ & $0.5 \pm 0.1$ \\
C4ALC $(n=5)$ & $3.9 \pm 0.5$ & $1.2 \pm 0.2$ & $0.3 \pm 0.2$ \\
D4 $(n=5)$ & $10.2 \pm 0.9^{*}$ & $4.7 \pm 0.5^{*}$ & $3.0 \pm 0.2^{*}$ \\
D4ALC $(n=5)$ & $3.9 \pm 0.6$ & $0.9 \pm 0.4$ & $0.8 \pm 0.3$ \\
8 mo & & & \\
C8 $(n=8)$ & $2.4 \pm 0.4$ & $1.3 \pm 0.3$ & $0.9 \pm 0.2$ \\
C8ALC $(n=8)$ & $2.6 \pm 0.3$ & $1.0 \pm 0.2$ & $0.5 \pm 0.2$ \\
D8 $(n=9)$ & $5.6 \pm 0.5^{*}$ & $3.3 \pm 0.6^{\ddagger}$ & $2.3 \pm 0.5^{\ddagger}$ \\
D8ALC $(n=9)$ & $2.6 \pm 0.3$ & $1.5 \pm 0.2^{\S}$ & $2.3 \pm 0.4^{\ddagger}$ \\
D4-8ALC $(n=6)$ & $1.8 \pm 0.2$ & $1.6 \pm 0.3^{\S}$ & $3.3 \pm 0.4^{\ddagger}$ \\
& & &
\end{tabular}

The earliest detectable nodal change consists of paranodal axonal swelling which is followed by paranodal demyelination. These abnormalities are repaired by remyelination of the nodal and paranodal areas, resulting in short, thinly myelinated, intercalated internodes. Designation of animal groups is the same as in Table I. $* P<0.001$ vs all groups; ${ }^{\ddagger} P<$ 0.001 vs $\mathrm{C} 8$ and C8ALC groups; ${ }^{\S} P<0.006$ vs D8. treated diabetic rats (Fig. $2 a$ ), which, following ALC treatment, showed a further twofold $(P<0.001)$ increase (Fig. $2 a)$. ALC treatment of nondiabetic control rats had no effect on any of the teased fiber parameters.

$8 \mathrm{mo}$. The frequency of structurally normal fibers was decreased by $27 \%(P<0.001)$ in nontreated diabetic rats compared to age-matched control rats. ALC treatment significantly protected $(P<0.001)$ and corrected $(P<0.001)$ normalcy of myelinated fibers in diabetic rats treated for 8 and 4 mo, respectively (Fig. $2 b$ ). These effects were reflected in significant decreases in the frequencies of both axonal $(P<0.001)$ and nodal $(P<0.004)$ degenerative changes in both groups of rats treated with ALC. Axonal atrophy assessed by excessive myelin wrinkling was reduced by $75 \%(P<0.001)$ and significantly corrected $(P<0.001)$ when compared to 8 and 4 mo nontreated diabetic rats respectively (Table V). Wallerian degeneration in diabetic rats was not affected by ALC treatment (Table V). Both paranodal swelling and demyelination were fully prevented or corrected by ALC treatment (Table VI); whereas, the frequency of the reparative nodal change, intercalated internodes, was not altered by ALC treatment. The already increased frequency of regenerating fibers $(P<0.001)$ in nontreated diabetic rats showed a further twofold increase after both 8 and 4 mo treatments with ALC (Fig. $2 b ; P<0.001$ for both). ALC treatment did not demonstrate any effects on teased-fiber morphologies in control rats.

\section{Discussion}

In the present studies, we have demonstrated both short- and long-term beneficial effects of ALC treatment on the metabolic, functional, and structural abnormalities characterizing neuropathy in the spontaneously diabetic BB/W rat. In addition, we have for the first time demonstrated a beneficial effect of ALC treatment on already established neuropathy in this animal model, with reversal of both functional and structural abnormalities.

Despite these beneficial effects in the prevention and reversal of neuropathy in the $\mathrm{BB} / \mathrm{W}$ rat, in neither situation did ALC treatment achieve complete normalization, suggesting that mechanisms in addition to deficiencies in carnitine or its short-chain esters must be operable in the pathogenesis of diabetic neuropathy. From the present data, it is clear that ALC does not act via inhibition of the polyol pathway, since no effect on sorbitol or fructose levels was demonstrated. However, the biochemical data indicate that ALC supplementation has an early beneficial effect on $\mathrm{Na}^{+} / \mathrm{K}^{+}$-ATPase activity, suggesting that depletion of acetyl-L-carnitine effects $\mathrm{Na}^{+} / \mathrm{K}^{+}$-ATPase activity in peripheral nerve fiber via metabolic pathways alternate to the polyol pathway.

Carnitine and its acetyl ester acetyl-L-carnitine are known to promote the transport of cytosolic long-chain fatty acids into mitochondria for $\beta$-oxidation (15-17), thereby providing mitochondrial energy. We have recently demonstrated that ALC treatment of acutely diabetic streptozotosin rats prevents transient nerve myoinositol depletion, probably by correcting PKC-regulated Na-dependent myoinositol transport and $\mathrm{Na}^{+}$/ $\mathrm{K}^{+}$-ATPase activity (16). However, as shown in the present study, the lack of a significant effect of 4 mo of diabetes or ALC treatment on nerve myoinositol levels in the presence of decreased and corrected $\mathrm{Na}^{+} / \mathrm{K}^{+}$-ATPase activities, respectively, suggests that metabolic aberrations, alternate to myo- 
inositol depletion, are influencing $\mathrm{Na}^{+} / \mathrm{K}^{+}$-ATPase activity at this stage of neuropathy. It has been demonstrated that accumulation of long-chain fatty acids and their esters in diabetic nerve may adversely effect phosphoinositide and PKC metabolism with secondary effects on $\mathrm{Na}^{+} / \mathrm{K}^{+}$-ATPase activities (22, 23). ALC repletion may therefore improve the acute metabolic nerve conduction defect by promoting $\beta$-oxidation of fatty acids, thereby eliminating their toxic accumulation in peripheral nerve and simultaneously correct $\mathrm{Na}^{+} / \mathrm{K}^{+}$-ATPase activity, without invoking a myoinositol-mediated mechanism.

In the present long-term studies, we failed to demonstrate an effect of diabetes on $\mathrm{Na}^{+} / \mathrm{K}^{+}$-ATPase, nor did ALC treatment alter the $\mathrm{Na}^{+} / \mathrm{K}^{+}$-ATPase activity in 8 mo diabetic rats. These findings suggest that in chronic diabetic neuropathy, myoinositol depletion and decreased $\mathrm{Na}^{+} / \mathrm{K}^{+}$-ATPase activity are of lesser pathogenetic significance, and that alternate mechanisms may become more prominent in sustaining the progressive degenerative process.

It is interesting to note that in 8 mo diabetic animals, nerve glucose levels were double, sorbitol levels approximately half, and fructose levels more than three times those of 4 mo diabetic animals. These findings suggest decreased aldose reductase activity and increased sorbitol dehydrogenase activity in the polyol pathway with increasing age and/or increasing duration of diabetes. Aldose reductase activity increases up to puberty and shows a progressive decline in senescent nondiabetic rats (Yagihashi, S., and A.A.F. Sima, unpublished data). Increased nerve fructose levels with duration of diabetes suggests increased shunting of sorbitol through the second step of the polyol pathway, perturbing further the redox coupling of $\mathrm{NADH} / \mathrm{NAD}^{+}$and therefore enhancing the effect of pseudohypoxia (10). In addition, since fructose is a potent glycator of proteins $(9,24)$, the threefold increase in fructose in 8 mo diabetic rats may suggest an increasing role for advanced glycation end products and associated generation of oxygen free radicals (11) in the pathophysiology of chronic diabetic neuropathy.

In recent years, it has become evident that decreased endoneurial blood flow, mediated by aberrations of vasoactive eicosanoids and NO, may play important pathogenetic roles in diabetic neuropathy $(8,11,13,25)$. An abnormality in the conversion of $\gamma$-linoleic acid to $\gamma$-linolenic acid effects the synthesis of arachidonate and vasoactive PGs (26), thereby promoting ischemia and further perturbing $\mathrm{Na}^{+} / \mathrm{K}^{+}$-ATPase activity. Indeed, treatment of diabetic rats with $\gamma$-linolenic acid prevents nerve conduction slowing and normalizes peripheral nerve blood flow (26).

The present data demonstrating a correction of the $\gamma$-linolenic acid-derived $\mathrm{PGE}_{1}$ in peripheral nerve after ALC treatment is in keeping with this notion, and may indeed contribute to increased blood flow (12), and hence to the beneficial effects on nerve conduction velocity and neuropathologic changes reported in the present studies. On the other hand, it is more difficult to reconcile the lack of an effect on the arachidonic acid-derived 6-keto $\mathrm{PGF}_{1 \alpha}$ and $\mathrm{PGE}_{2}$ in view of the beneficial effect ALC treatment has on arachidonic acid metabolism $(14,17)$. The decrease in both 6-keto $\mathrm{PGF}_{1 \alpha}$ and $\mathrm{PGE}_{2}$ in ALC-treated control rats may suggest that ALC treatment enhances the metabolism of these PGs via their $\beta$-oxidation-dependent transformation into the water soluble tetranor compounds excreted by the kidney (27). If this notion is correct, it may represent an additional, potentially beneficial, effect of ALC, since it has been suggested that 6-keto $\mathrm{PGF}_{1 \alpha}$ and $\mathrm{PGE}_{2}$ have dilatory effects on large vessels but a contractile effect on small vessels (28), in contrast to $\mathrm{PGE}_{1}$.

It therefore appears that ALC prevents the acute $\mathrm{Na}^{+} / \mathrm{K}^{+}$ATPase defect in diabetic nerve without involving the polyol pathway and myoinositol metabolism, resulting in a preventive effect on the acute nerve conduction slowing similar to that seen following short-term treatment with an aldose reductase inhibitor in the diabetic $\mathrm{BB} / \mathrm{W}$ rat $(7,19)$. In addition, the present data demonstrate a beneficial effect on vasoactive PGs, suggesting improved nerve blood flow and hence further benefiting nerve conduction and structure. It is therefore not surprising that ALC administration showed positive effects on the structural peripheral nerve abnormalities similar to those reported following long-term aldose reductase inhibitor treatments (19), preventing both axonal and nodal degenerative changes characteristic of diabetic neuropathy $(19,20)$. Furthermore, the present data have for the first time demonstrated reversal of functional and repair of structural changes after ALC administration, underpinning its corrective effects on underlying metabolic abnormalities. Although the preventive and corrective effects of ALC treatment on nerve function and pathology were not complete, they appeared to substantially attenuate the development of more severe chronic changes.

A striking finding in the current studies was the beneficial effect of ALC administration on nerve fiber regeneration, similar to the long-term effects of aldose reductase inhibitors (19). The twofold increase in regenerating fibers in 8 mo treated diabetic rats was reflected by an increase in fiber density, decreased mean fiber size, and increased axon/myelin ratio, all consistent with the addition of small thinly myelinated regenerating fibers. These findings were less evident in D4-8ALC rats, probably reflecting a less pronounced effect after shorter treatment. The promoting effect of ALC on nerve fiber regeneration may in part be explained by the corrective effect of ALC on underlying dysmetabolism, but may also be secondary to the enhancing effect ALC has on the expression of nerve growth factor receptors and nerve growth factor levels (29).

In summary, our study demonstrates significant, although not complete, prevention and correction of the metabolic, functional, and structural abnormalities characterizing the neuropathy in the diabetic $\mathrm{BB} / \mathrm{W}$ rat after ALC administration. In addition, the same treatment showed a promoting effect on suppressed nerve fiber regeneration in diabetic rats. Although the nature of the underlying metabolic aberrations corrected by ALC administration are not known in detail, it appears that normalization of $\beta$-oxidation of fatty acids and elimination of excessive accumulation of toxic long-chain fatty acids in diabetic nerve would favor the early normalization of $\mathrm{Na}^{+} / \mathrm{K}^{+}$-ATPase activity. In addition, ALC treatment appears to have a sustained beneficial effect on vasoactive PGs, possibly counteracting the deleterious effects of decreased endoneurial blood flow in diabetic nerve.

We therefore conclude that ALC administration has beneficial effects on the prevention and correction of the long-term sequelae of diabetic neuropathy in the spontaneously diabetic $\mathrm{BB} / \mathrm{W}$ rat, and may therefore be a suitable compound for the treatment of this complication in man.

\section{Acknowledgments}

These studies were supported by a grant from Sigma-Tau, Gaithersburg, MD; U.S. Public Health Service research grants RO1-DK43884 
(A.A.F. Sima), RO1-DK38304 (D.A. Greene), and the Michigan Diabetes Research and Training Center P60-DK20572 (D.A.Greene, A.A.F. Sima).

The authors are indebted to Douglas Heady for assistance in the quality control studies for PG analysis, to Rhonda Lightle for technical assistance, and to Elaine Moore for preparing the manuscript.

\section{References}

1. Dyck, P.J., J.L. Karnes, P. O’Brien, H. Okazaki, A. Lais, and J. Englestad. 1986. The spatial distribution of fiber loss in diabetic neuropathy suggests ischemia. Ann. Neurol. 19:440-449.

2. Sima, A.A.F., V. Bril, V. Nathaniel, T.A. McEwen, M. Brown, S.A. Lattimer, and D.A. Greene. 1988. Regeneration and repair of myelinated fibers in sural nerve biopsies from patients with diabetic neuropathy treated with an aldose reductase inhibitor. N. Engl. J. Med. 319:548-555.

3. Sima, A.A.F., V. Nathaniel, V. Bril, T.A.J. McEwen, and D.A. Greene. 1988. Histopathological heterogeneity of neuropathy in insulin-dependent and non-insulin-dependent diabetes, and demonstration of axo-glial dysjunction in human diabetic neuropathy. J. Clin. Invest. 81:349-364.

4. Johnson, P.C., S.C. Doll, and D.W. Cromey. 1986. Pathogenesis of diabetic neuropathy. Ann. Neurol. 19:450-457.

5. Sima, A.A.F., M. Bouchier, and H. Christensen. 1983. Axonal atrophy in sensory nerves of the diabetic BB-Wistar rat, a possible early correlate of human diabetic neuropathy. Ann. Neurol. 13:264-272.

6. Medori, R., L. Autilio-Gambetti, H. Jevidi, and P. Gambetti. 1988. Changes in axon size and slow axonal transport are related in experimental diabetic neuropathy. Neurology. 38:597-601.

7. Sima, A.A.F., S.A. Lattimer, S. Yagihashi, and D.A. Greene. 1986. Axoglial dysjunction: a novel structural lesion that accounts for poorly reversible slowing of nerve conduction in the spontaneously diabetic bio-breeding rat. $J$. Clin. Invest. 77:474-484.

8. Greene, D.A., A.A.F. Sima, M. Stevens, E. Feldman, P. Killen, D. Henry, T. Thomas, J. Dannenberg, and S.A. Lattimer. 1993. Aldose reductase inhibitors: an approach to the treatment of the nerve damage of diabetic neuropathy. Diabetes Metab. Rev. 9:189-217.

9. Dvornik, D. 1987. Aldose reductase inhibition. An approach to the prevention of diabetic complications. Biomedical Information Corporation (McGraw-Hill), New York. 222-323.

10. Williamson, J.R., K. Chang, M. Frangos, K.S. Hasan, Y. Ido, T. Kawamura, J.R. Nyengaard, M. van den Enden, C. Kilo, and R.G. Tilton. 1993. Hyperglycemic pseudohypoxia and diabetic complications. Diabetes. 42:801-813.

11. Cameron, N.E., and M.A. Cotter. 1994. The relationship of vascular changes to metabolic factors in diabetes mellitus and their role in the development of peripheral nerve complications. Diabetes Metab. Rev. 10:189-224.

12. Cotter, M.A., K.C. Dines, and N.E. Cameron. 1993. Comparison of the effect of evening primrose oil and the putative active triglyceride, di-linolein mono- $\gamma$-linolenate (DLMG), on sciatic nerve conduction velocity in diabetic rats: dose response relationship and modulation of endoneurial blood flow. $D i$ abetic Med. 10(Suppl. 3):67.

13. Sonobe, M., H. Yasuda, T. Hisanaga, K. Maeda, M. Yamashita, T. Kawabata, R. Kikkawa, Y. Taniguchi, and Y. Shigeta. 1991. Amelioration of nerve $\mathrm{Na}^{+}-\mathrm{K}^{+}$-ATPase activity independently of myo-inositol level by PGE1 analogue OP-1206- $\alpha-\mathrm{CD}$ in streptozotocin-induced diabetic rats. Diabetes. 40: 726-730.

14. Williamson, J.R., and E. Arrigoni-Martelli. 1992. The roles of glucoseinduced metabolic hypoxia and imbalances in carnitine metabolism in mediating diabetes-induced vascular dysfunction. Int. J. Clin. Pharmacol. Res. 12:247252

15. Ido, Y., J. McHowat, K.C. Chang, E. Arrigoni-Martelli, Z. Orfalian, C. Kilo, P.B. Corr, and J.R. Williamson. 1994. Neural dysfunction and metabolic imbalances in diabetic rats. Prevention by acetyl-L-carnitine. Diabetes. 43:14691477.

16. Stevens, M., S. Lattimer, E. Feldman, A.A.F. Sima, and D.A. Greene. 1995. Correction of nerve conduction slowing, nerve myoinositol but not sorbitol by acetyl-L-carnitine in the streptozotocin-diabetic rat. Diabetes. 44(Suppl. 1):66a Abstr.

17. Lowitt, S., J.I. Malone, A. Salem, W.M. Kozak, and Z. Orfalian. 1993. Acetyl-L-carnitine corrects electroretinographic deficits in experimental diabetes. Diabetes. 42:1115-1118.

18. Sima, A.A.F., and T. Brismar. 1985. Reversible diabetic nerve dysfunction. Structural correlates to electrophysiological abnormalities. Ann. Neurol. 18:21-29.

19. Sima, A.A.F., A. Prashar, W.-X. Zhang, S. Chakrabarti, and D.A. Greene. 1990. Preventive effect of long-term aldose reductase inhibition (ponalrestat) on nerve conduction and sural nerve structure in the spontaneously diabetic bio-breeding rat. J. Clin. Invest. 85:1410-1420.

20. Sima, A.A.F. 1988. Natural history of structural and functional alterations in diabetic BB-rat peripheral nerve. In Frontiers in Diabetes Research: Lessons from Animal Diabetes II. A.E. Renold and E. Shafrir, editors. John Libbey and Company, London. 474-476.

21. Stevens, M.J., D.N. Henry, T.P. Thomas, P.D. Killen, and D.A. Greene. 1993. Aldose reductase gene expression and osmotic dysregulation in cultured human retinal pigment epithelial cells. Am. J. Physiol. 265:E228-E238.

22. Pugliese, G., R.G. Tilton, and J.R. Williamson. 1991. Glucose-induced metabolic imbalances in the pathogenesis of diabetic vascular disease. Diabetes Metab. Rev. 7:35-59.

23. Corr, P.B., R.W. Gross, and B.E. Sobel. 1984. Amphipathic metabolites and membrane dysfunction in ischemia myocardium. Circ. Res. 55:135-144.

24. Bunn, H.F., and P.J. Higgins. 1981. Reaction of monosaccharides with protein. Possible evolutionary significances. Science (Wash. DC). 213:222-224.

25. Cotter, M.A., K.C. Dines, and N.E. Cameron. 1993. Prevention and reversal of motor and sensory peripheral nerve conduction abnormalities in streptozotocin-diabetic rats by the prostacyclin analogue iloprost. NaunynSchmiedeberg's Arch. of Pharmacol. 347:534-540.

26. Cameron, N.E., M.A. Cotter, and S. Robertson. 1991. Essential fatty acid diet supplementation. Effects on peripheral nerve and skeletal muscle function and capillarization in streptozotocin-induced diabetic rats. Diabetes. 40:532-539.

27. Lee, B.J. 1981. The prostaglandins. In Textbook of Endocrinology. R.H. Williams, editor. 6th Edition. W.B. Saunders Co., Philadelphia. 1047-1063.

28. Rogers, S.P., and R.G. Larkins. 1982. Production of 6-oxo-prostaglandin $\mathrm{F}_{1 \alpha}$ and prostaglandin $\mathrm{E}_{2}$ by isolated glomeruli from normal and diabetic rats. Br. Med. J. 284:1214-1217.

29. Taglialatela, G., L. Angelucci, M.T. Ramacci, K. Werrbach-Perez, G.R. Jackson, and J.R. Perez-Polo. 1991. Acetyl-L-carnitine enhances the response of PC12 cells to nerve growth factor. Dev. Brain Res. 59:221-230. 